\title{
TU/e EN⿴HONE

\section{Hankel Iterative Learning Control for residual vibration suppression with MIMO flexible structure experiments}

\section{Citation for published version (APA):}

Wijdeven, van de, J. J. M., \& Bosgra, O. H. (2007). Hankel Iterative Learning Control for residual vibration suppression with MIMO flexible structure experiments. In Proceedings of the 2007 American Control Conference (ACC 2007) 9-13 July 2007, New York, New York, USA (pp. 4993-4998). Institute of Electrical and Electronics Engineers. https://doi.org/10.1109/ACC.2007.4282208

DOI:

10.1109/ACC. 2007.4282208

Document status and date:

Published: 01/01/2007

\section{Document Version:}

Publisher's PDF, also known as Version of Record (includes final page, issue and volume numbers)

\section{Please check the document version of this publication:}

- A submitted manuscript is the version of the article upon submission and before peer-review. There can be important differences between the submitted version and the official published version of record. People interested in the research are advised to contact the author for the final version of the publication, or visit the $\mathrm{DOI}$ to the publisher's website.

- The final author version and the galley proof are versions of the publication after peer review.

- The final published version features the final layout of the paper including the volume, issue and page numbers.

Link to publication

\section{General rights}

Copyright and moral rights for the publications made accessible in the public portal are retained by the authors and/or other copyright owners and it is a condition of accessing publications that users recognise and abide by the legal requirements associated with these rights.

- Users may download and print one copy of any publication from the public portal for the purpose of private study or research.

- You may not further distribute the material or use it for any profit-making activity or commercial gain

- You may freely distribute the URL identifying the publication in the public portal.

If the publication is distributed under the terms of Article 25fa of the Dutch Copyright Act, indicated by the "Taverne" license above, please follow below link for the End User Agreement:

www.tue.nl/taverne

Take down policy

If you believe that this document breaches copyright please contact us at:

openaccess@tue.nl

providing details and we will investigate your claim. 


\section{Hankel Iterative Learning Control for residual vibration suppression with MIMO flexible structure experiments}

\author{
Jeroen van de Wijdeven \\ Technische Universiteit Eindhoven \\ Dept. of Mechanical Engineering \\ P.O. Box 513, $5600 \mathrm{MB}$ Eindhoven \\ The Netherlands \\ J.J.M.v.d.Wijdeven@tue.nl
}

\author{
Okko Bosgra \\ Technische Universiteit Eindhoven \\ Dept. of Mechanical Engineering \\ P.O. Box 513, 5600 MB Eindhoven \\ The Netherlands \\ O.H.Bosgra@tue.nl
}

\begin{abstract}
In this paper, we consider residual vibration suppression in flexible structures performing a point-to-point motion, based on Hankel ILC. Initially, design freedom in Hankel ILC is discussed, including different choices for the actuation time window and the observation time window. Subsequently, a three input three output flexible beam is presented as an experimental setup for Hankel ILC. The different practical and theoretical issues related to implementation of Hankel ILC on the setup are discussed extensively. Thereby, versatility in the choice for the time windows is shown to be essential for a successful implementation. Experimental results illustrate the capability of Hankel ILC to suppress the residual vibrations in the flexible beam.
\end{abstract}

\section{INTRODUCTION}

In this paper, we address the issue of residual vibration suppression of multi input multi output (MIMO) flexible structures performing a point-to-point motion. The challenge in residual vibration suppression is to find a command signal actuating the system during the point-to-point motion, resulting in the system to be at rest after arrival at the desired position.

In existing literature, input shaping techniques are proposed to handle these residual vibrations, e.g., [1-3]. Recently though, an alterative technique for residual vibration suppression is presented based on Iterative Learning Control (ILC), [4-6]. ILC is a control strategy used to iteratively improve the performance of a repeated batch process by updating the command signal from one experiment (trial) to the next. This command signal is updated using measurement data from previous trials, i.e., by learning from previous trials. Treatment of different topics in ILC can be found in, e.g., [7-10].

Originally, ILC is used to let a system follow a given reference, e.g., [11-13]. In [4-6] however, actuation and observation time windows are introduced to separate the actuation and observation time intervals, thereby making ILC capable of handling residual vibrations in systems performing point-to-point motions. Due to the choice of nonoverlapping adjacent time windows, ILC applied to pointto-point problems is referred to as Hankel ILC. In [46], different Hankel ILC control strategies are derived and different alternatives for the time windows are presented. What is missing in [4-6] though, are extensive experimental results of Hankel ILC to support the theory.
The contribution of this paper is threefold. First of all, we will illustrate the concept of Hankel ILC on a relatively complex MIMO flexible structure and show, by means of experiments, that residual vibrations after a point-to-point motion can indeed be suppressed. Second, we will show the consequence of applying different actuation and observation time windows on the attainable performance of ILC. And third, we will demonstrate that Hankel ILC incorporates the possibility to manipulate the command signal form.

The outline of this paper is as follows. In Section II, the applied ILC notations are introduced, together with theoretical results of Hankel ILC. In Section III, the flexible beam setup is introduced and modified to be suitable for ILC. Subsequently, in Section IV, the results of ILC and Hankel ILC on the flexible beam are presented. This paper ends with concluding remarks in Section V.

\section{HANKEL ILC}

In subsection II-A, the different signals and systems present in (Hankel) ILC are presented. This is followed by a brief discussion on actuation and observation time windows in subsection II-B. Finally, in subsection II-C two Hankel ILC control strategies are presented.

\section{A. Iterative Learning Control}

The ILC control problem in this paper is studied in the lifted setting, [13-15]. In this setting, the behavior of a discrete-time linear time invariant (LTI) system $J$ during a trial is represented by its convolution matrix. This matrix contains the systems impulse response data $H(t)$ for time $t=0,1, \cdots, N-1$, with $N$ the total number of samples in a trial:

$$
J=\left[\begin{array}{ccc}
H(0) & & 0 \\
\vdots & \ddots & \\
H(N-1) & \cdots & H(0)
\end{array}\right] .
$$

For MIMO systems, the impulse response $H(t)$ contains the impulse response from each input to each output. Given a system with $q_{i}$ inputs and $q_{o}$ outputs, $H(t)$ is represented by

$$
H(t)=\left[\begin{array}{ccc}
H^{11}(t) & \cdots & H^{1 q_{i}}(t) \\
\vdots & & \vdots \\
H^{q_{o}}(t) & \cdots & H^{q_{o} q_{i}}(t)
\end{array}\right],
$$


with $H^{i j}(t)$ the impulse response from input $j$ to output $i$. With $H(t)$ of (2), matrix $J \in \mathbb{R}^{N q_{o} \times N q_{i}}$ is a lower triangular matrix with a block Toeplitz structure.

The system matrix $J$ maps an input vector $f_{k}$ to an output vector $y_{k}$, i.e., $y_{k}=J f_{k}$, with $k$ the trial number. The vectors correspond to the lifted notation of time signals during a trial,

$$
\begin{aligned}
f_{k}= & {\left[\begin{array}{llll}
f_{k}^{T}(0) & f_{k}^{T}(1) & \cdots & f_{k}^{T}(N-1)
\end{array}\right]^{T} } \\
y_{k}= & {\left[\begin{array}{llll}
y_{k}^{T}(0) & y_{k}^{T}(1) & \cdots & y_{k}^{T}(N-1)
\end{array}\right]^{T}, } \\
\text { with } \quad & f_{k}^{T}(t)=\left[\begin{array}{lll}
f_{k}^{1}(t) & \cdots & f_{k}^{q_{i}}(t)
\end{array}\right] \\
\text { and } \quad & y_{k}^{T}(t)=\left[\begin{array}{lll}
y_{k}^{1}(t) & \cdots & y_{k}^{q_{o}}(t)
\end{array}\right] .
\end{aligned}
$$

The ILC control structure used in this paper is shown in Fig. 1, [5]. The system matrices $L_{L}$ and $L_{R}$ correspond to the "left" and "right" ILC controller matrices, $w^{-1} I$ to the one trial delay operator, $W_{i}$ and $W_{o}$ to the actuation and observation time windows respectively, and $J_{H}$ to the time weighted system $J_{H}=W_{o} J W_{i}$. The signal $\tilde{f}_{k}$ in Fig. 1 represents the input of $J_{H}, \tilde{y}_{k}$ the output of $J_{H}, r$ the reference signal to be followed, $\tilde{e}_{k}$ the error $\tilde{e}_{k}=W_{o}\left(r-y_{k}\right)$, and $u_{k}$ the trial domain state with $u_{0}=0$.

The trial domain dynamics corresponding to the control scheme of Fig. 1 are:

$$
\begin{aligned}
u_{k+1} & =u_{k}+L_{L} W_{o} e_{k} \quad \text { with } e_{k}=r-y_{k} \\
f_{k} & =W_{i} L_{R} u_{k} \\
u_{k+1} & =\left(I-L_{L} J_{H} L_{R}\right) u_{k}+L_{L} W_{o} r .
\end{aligned}
$$

While implementation of ILC on an experimental setup requires the use of (5) and (6), (7) is essential in the stability analysis of the ILC controlled system. Asymptotic stability of (7) is guaranteed if and only if $\rho\left(I-L_{L} J_{H} L_{R}\right)<1$, i.e., if $\max \left(\left|\lambda_{i}\left(I-L_{L} J_{H} L_{R}\right)\right|\right)<1$, with $\lambda_{i}(*)$ the $i^{t h}$ eigenvalue of $*$. For more stability results, see [5], [6].

\section{B. Actuation and observation time windows}

The actuation time window $W_{i}$ and observation time window $W_{o}$ can be used to separate actuation and observation time intervals during a trial, thereby making ILC capable of handling residual vibrations in systems preforming a pointto-point motion, [5]. A consequence of introducing $W_{i}$ is that outside the actuation interval, $f_{k}$ can not be manipulated by Hankel ILC. A consequence of introducing $W_{o}$ is, that errors $e_{k}$ outside the observation time interval are not compensated for by Hankel ILC.

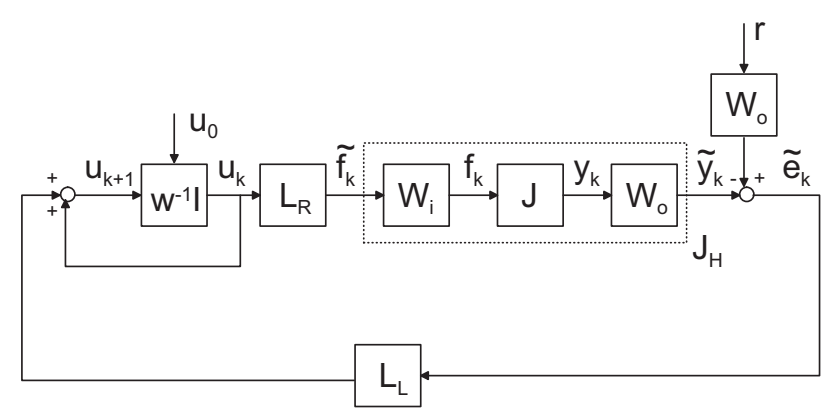

Fig. 1. General ILC control structure.
In this paper, we separate actuation and observation as given in Fig. 2. The actuation interval spans $t=\left\{m_{1}, m_{1}+1, \cdots, m_{2}\right\}$, the observation interval $t=$ $\left\{n_{1}, n_{1}+1, \cdots, n_{2}\right\}$, with $n_{1}:=m_{2}+1$. Hence the total number of samples in the actuation and observation time intervals equals $m:=m_{2}-m_{1}+1$ and $n:=n_{2}-n_{1}+1$ respectively.

While for a standard ILC problem $W_{i}=I_{N q_{i} \times N q_{i}}$ and $W_{o}=I_{N q_{o} \times N q_{o}}$, for the Hankel ILC problem illustrated by Fig. $2 W_{i}$ and $W_{o}$ equal

$$
\begin{aligned}
W_{i} & =\left[\begin{array}{lll}
0_{m q_{i} \times\left(m_{1}-1\right) q_{i}} & I_{m q_{i}} & 0_{m q_{i} \times\left(N-m_{2}\right) q_{i}}
\end{array}\right]^{T} \\
W_{o} & =\left[\begin{array}{ll}
0_{n q_{o} \times(N-n) q_{0}} & I_{n q_{o}}
\end{array}\right] .
\end{aligned}
$$

\section{Hankel ILC controller design}

Hankel ILC controller design, i.e., design of $L_{L}$ and $L_{R}$, is based on the time weighted system $J_{H}$. To find expressions for $L_{L}$ and $L_{R}$, we first rewrite $J_{H} \in \mathbb{R}^{n q_{o} \times m q_{i}}$, with $\operatorname{rank}\left(J_{H}\right)=: p \leq \min \left(n q_{o}, m q_{i}\right)$, using the non-unique full rank decomposition (10).

$$
J_{H}=J_{L} J_{R} \text {, with } J_{L} \in \mathbb{R}^{n q_{o} \times p} \text { and } J_{R} \in^{p \times m q_{i}} .
$$

In this paper, explicit expressions for $J_{L}$ and $J_{R}$ are found by using the Singular Value Decomposition (SVD) of $J_{H}$, see Appendix,

$$
J_{L}=U_{1}, \quad J_{R}=\Sigma_{1} V_{1}^{T} .
$$

Based on (11) and [4], [6], $L_{L}$ and $L_{R}$ are expressed by (12) and (13) respectively.

$$
\begin{aligned}
L_{L} & =J_{L}^{\dagger}:=\left(J_{L}^{T} J_{L}\right)^{-1} J_{L}^{T}=U_{1}^{T} \\
L_{R} & =X_{1}+\left(I_{m q_{i}}-J_{R}^{\dagger} J_{R}\right) Y \\
& =X_{1}+V_{2} V_{2}^{T} Y, \\
X_{1} & =\gamma J_{R}^{T}\left(J_{R} J_{R}^{T}+\beta I_{p}\right)^{-1} \\
& =\gamma V_{1} \Sigma_{1}\left(\Sigma_{1}^{2}+\beta I_{p}\right)^{-1},
\end{aligned}
$$

with $Y \in \mathbb{R}^{m q_{i} \times p}$ arbitrary, and $\beta \geq 0$ and $0<\gamma<2$ controller parameters. In [5], [6], it is shown that the ILC controlled system of Fig. 1, with $L_{L}$ and $L_{R}$ given by (12) and (13):

- is asymptotically stable, due to $X_{1}$.

- achieves optimal performance, i.e., $\min \lim _{k \rightarrow \infty}\left\|\tilde{e}_{k}\right\|$ in an appropriate norm, through $L_{L}$.

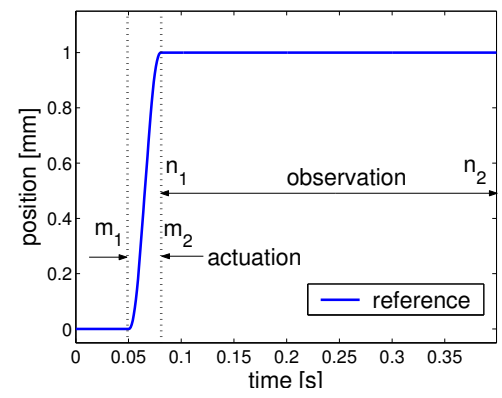

Fig. 2. Reference signal with separated actuation and observation time interval. $m_{1}, m_{2}, n_{1}$, and $n_{2}$ are sample instants, with $n_{1}=m_{2}+1$. 
- can be used to manipulate the signal form of the command signal $\tilde{f}_{k}$, using $Y$.

The Hankel ILC controllers studied in this paper use $L_{L}$ and $X_{1}$ as given in (12) and (14) respectively. The difference between the controllers is found in the expression for $Y$.

The first $L_{R}$ controller uses an analytical expression for $Y$ derived from the optimization problem $\min \tilde{f}_{\infty}^{T} W \tilde{f}_{\infty}$. Here, $\tilde{f}_{\infty}$ equals $\tilde{f}_{k}$ for $k \rightarrow \infty$ and $W \in \mathbb{R}^{m q_{i} \times m q_{i}}$ is a weighting matrix penalizing the entries in $\tilde{f}_{\infty}$. The resulting $L_{R}$ controller is given by [4], [6]:

$$
L_{R}=\left(I_{m q_{i}}-V_{2}\left(V_{2}^{T} W V_{2}\right)^{-1} V_{2}^{T} W\right) X_{1} .
$$

The second $L_{R}$ controller focusses on minimizing the maximum command signal amplitude during the actuation time interval $f_{k+1, a c t}$ :

$$
\begin{aligned}
& f_{k+1}=W_{i} \tilde{f}_{k+1}, \quad f_{k+1, a c t}=W_{a c t} f_{k+1} \\
& W_{a c t}=\left[\begin{array}{lll}
0_{m q_{i} \times\left(m_{1}-1\right) q_{i}} & I_{m q_{i}} & 0_{m q_{i} \times\left(N-m_{2}\right) q_{i}}
\end{array}\right] .
\end{aligned}
$$

With $f_{k+1, a c t}=W_{a c t} W_{i}\left(X_{1} u_{k+1}+V_{2} V_{2}^{T} Y u_{k+1}\right)$, the corresponding optimization problem equals [6]:

$$
\begin{gathered}
\min _{\theta_{k+1}}\left|W_{a c t} W_{i}\left(X_{1} u_{k+1}+V_{2} V_{2}^{T} \theta_{k+1}\right)\right|, \quad \text { subject to } \\
\left|f_{k+1, a c t}(t+1)-f_{k+1, a c t}(t)\right| \leq \Delta_{f}, \forall \quad t \in[1, m],
\end{gathered}
$$

with $\theta_{k+1}=Y u_{k+1} \in \mathbb{R}^{m q_{i} \times 1}$, and $\Delta_{f}>0$ an additional rate bound on $f_{k+1}$ in time domain.

The command signal for trial $k+1$ is now given by:

$$
f_{k+1}=W_{i}\left(X_{1} u_{k+1}+V_{2} V_{2}^{T} \theta_{k+1, o p t}\right),
$$

where $\theta_{k+1, o p t}$ is the minimizing solution of (17).

\section{EXPERIMENTAL SETUP}

The experimental setup used for Hankel ILC is presented in Fig. 3. The steel beam $(500 \mathrm{~mm} \times 20 \mathrm{~mm} \times 2 \mathrm{~mm})$ is fixed to the environment by five leaf springs, which remove four degrees of freedom (DOF). The two remaining DOFs consist of a translation in $x$ direction and rotation around $\varphi$.

The system is actuated by three current driven Lorentz' voice coil actuators. Two actuators are required to control the two DOFs, while the third actuator can be used to suppress flexible modes in the beam. The input of an actuator is provided by an amplifier (voltage-to-current converter) with an input voltage approximately proportional to the output

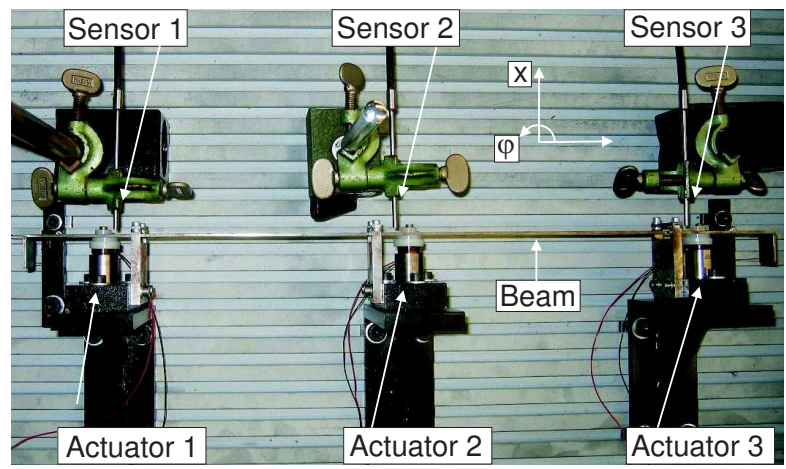

Fig. 3. The flexible beam setup. current. The input of the amplifier is limited to $[-2.5,2.5]$ volt.

The position of the beam is measured with fiberoptic sensors. These sensors perform non-contact measurements of the displacement of the beam by transmitting light and measuring the intensity of the reflected light. In our range of operation, the displacement-to-intensity ratio is approximately constant. The experimentally determined noise level, i.e., standard deviation of the measured output, is approximately $0.35 \mu \mathrm{m}$.

For control implementation on the flexible beam, we use a rapid prototyping environment. It consists of real-time hardware which is connected to the amplifiers and sensors, in combination with Matlab Simulink. In this paper, the experiments are performed with a sample time $T_{s}$ of $1 \mathrm{~ms}$.

For ILC to be implementable on the experimental setup, the ILC control problem and setup should satisfy the following conditions [7].

- A trial has a fixed and finite time span.

- The reference signal $r$ is known over the complete trial time interval.

- Repetition of initial time domain state for each trial, i.e., $x_{k}(0)=x_{0}$.

- Invariance of the system dynamics is ensured throughout all trials.

The reference signal applied to the system is given in Fig. 2 , thereby we satisfy the first two conditions. To meet the third condition, we have developed a homing procedure which brings the system within $0.4 \mu \mathrm{m}$ of the desired initial position.

The fourth condition has provided more difficulties. When repeatedly applying a pulse with an amplitude of 1 volt to the three actuators (20 trials with a trial length of 1 second), the three measured outputs vary much more than $0.4 \mu \mathrm{m}$, Table I second column. Without knowing the exact source for the non-repetitiveness of the outputs, spectral analysis of the outputs reveals that the non-repetitiveness is dominated by the low frequent rigid body frequencies.

TABLE I

REPETITIVENESS OF THE SYSTEMS DYNAMICS, (STANDARD DEVIATION IN $[\mu \mathrm{m}])$.

\begin{tabular}{|c|c|c|c|}
\hline noise & open loop & closed loop & closed loop with integrator \\
\hline 0.35 & 3.53 & 0.95 & 0.85 \\
\hline
\end{tabular}

To improve the repetitiveness of our system, we introduce feedback control into the time domain loop. With the variances in the outputs dominated by the two rigid body frequencies, we use time domain feedback control to control these two rigid body modes. The output $y^{1}$ and $y^{3}$ are first transformed to the rigid body coordinates $y^{1, r b}$ (translations) and $y^{2, r b}$ (rotation), using matrix $T_{y}$. Matrix $T_{y}$ is determined based on the geometry of the system, resulting in (19).

$$
T_{y}=\left[\begin{array}{cc}
\frac{1}{\sqrt{2}} & \frac{1}{\sqrt{2}} \\
\frac{1}{\sqrt{2}} & -\frac{1}{\sqrt{2}}
\end{array}\right] \text {. }
$$


In this coordinate system, a diagonal controller is designed consisting of a lead filter with a zero at $10 \mathrm{~Hz}$, a pole at 90 $\mathrm{Hz}$, and a gain of 0.15 , in series with a low pass filter with a cut-off frequency of $400 \mathrm{~Hz}$. Subsequently, the feedback controller outputs $u^{1, r b}$ and $u^{3, r b}$ are transformed back to $u^{1}$ and $u^{3}$, using matrix $T_{u}$. With the positions of the actuators and sensors approximately equal, we choose $T_{u}=T_{y}^{-1}=$ $T_{y}$.

The time domain closed loop system is shown in Fig. 4. Note that, since the second actuator and output are not used in the feedback loop, the system in Fig. 4 is partly open loop, partly closed loop. If we again apply the pulse of 1 volt to the three actuators, the repetitiveness of the output after 20 trials is significantly improved, Table I third column.

In an attempt to further improve the invariance of the dynamics, we introduce an integrator in each of the two diagonal entries with a gain of 0.5 . The result in repetitiveness is indeed improved, Table I fourth column. A closer look at the error signals of the closed loop system with and without the integrator reveals, however, that for the integrator case the non-repetitiveness during the transient time interval is larger, but that the steady state offset is smaller.

Looking at the results of Table I, the closed loop system including the integrator outperforms the closed loop system without the integrator. Based on this, and the fact that our reference signal contains relatively long constant outputs which require small steady state errors, we decide to perform ILC and Hankel ILC to the closed loop system with feedback control including the integrator action.

To apply ILC to the flexible beam, we require the impulse response of the system. This impulse response of the system is obtained by separately applying a pulse with an amplitude of 2 volt to each of the actuators and measure the response of all three outputs. These experiments are repeated 20 times for each actuator. Afterwards, the measurements are averaged and divided by 2 , to correct for the pulse amplitude. The resulting impulse response of the time domain system is presented in Fig. 5.

\section{EXPERIMENTAL RESULTS}

\section{A. Standard ILC}

To apply standard ILC to the setup, we first construct the convolution matrix $J$, (1), using the impulse response data of Fig. 5. Furthermore, we define $W_{i}=I_{N q_{i}}$ and $W_{o}=I_{N q_{o}}$ such that $J_{H}=J$. Now, $L_{L}$ and $L_{R}$ are designed based on the SVD of $J_{H}$, our choice $p=300$, and $W=I_{N q_{i}}$, resulting in $L_{L}$ of (12) and $L_{R}=\gamma V_{1} \Sigma_{1}\left(\Sigma_{1}^{2}+\beta I_{p}\right)^{-1}$.

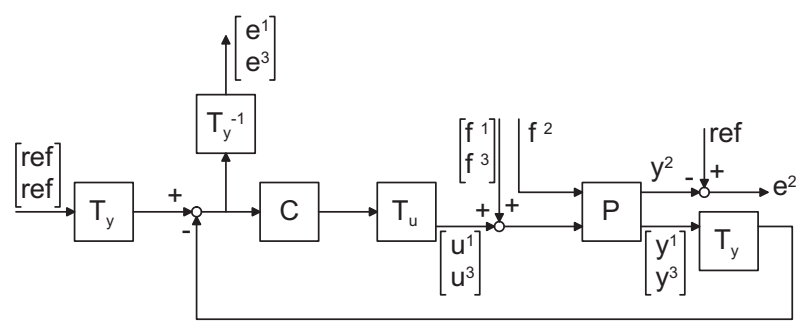

Fig. 4. Time domain closed loop system.
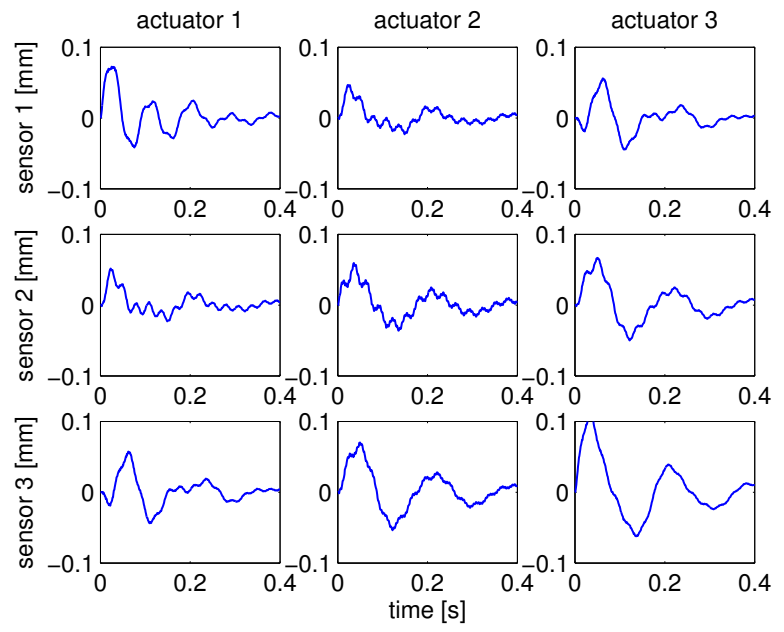

Fig. 5. Impulse response of the MIMO flexible beam, for $t=[0,0.4] \mathrm{s}$.

The values $\gamma=0.5$ and $\beta=0.01$ have been determined experimentally.

The error $e_{k}$ and command signal $f_{k}$ during trial $k=15$ are presented in Fig. 6. Though the error norm $\left\|e_{k}\right\|_{\infty}$ decreases as function of $k$, the command signal has an undesired signal form for $t \rightarrow 0.4 \mathrm{~s}$. Apparently, the ILC controller has problems of generating a constant command signal near the end of the trial. Increasing $p$ and/or decreasing $\beta$ does improve the effect near $t=0.4 \mathrm{~s}$, but results in nonsmoother command signals during the trial, or even in trial domain instability.

The problem with the command form can be explained by the fact that the system is type $0,[16]$. A solution is found by adding time domain integrators to the inputs of the system. For the control scheme of Fig. 1, these integrators can be incorporated in $W_{i}$, giving

$$
W_{i}:=\underbrace{\left[\begin{array}{ccc}
I_{q_{i}} & & 0 \\
\vdots & \ddots & \\
I_{q_{i}} & \cdots & I_{q_{i}}
\end{array}\right]}_{W_{i n t}}\left[\begin{array}{c}
0 \\
I_{m q_{i}} \\
0
\end{array}\right] .
$$
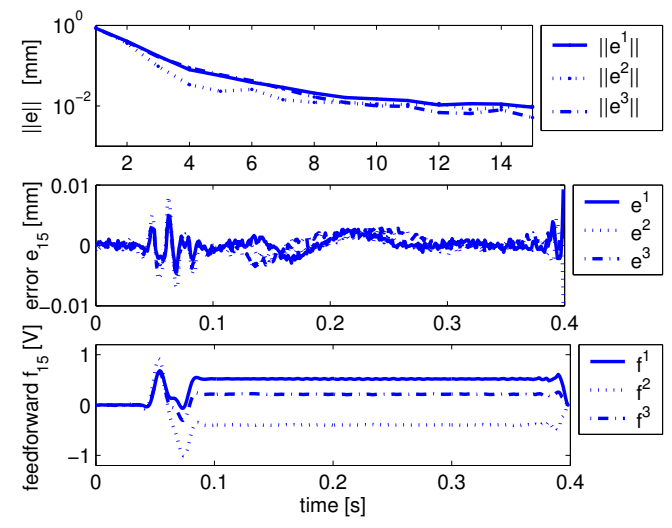

Fig. 6. Standard ILC. Top: Maximum absolute error as function of trial number. Center: Error signal during trial $k=15$. Bottom: Command signal during trial $k=15$. 
The ILC controllers are calculated with the SVD of $J \leftarrow J W_{\text {int }}$. The error and command signal results during trial 30 are shown in Fig. 7. Now, the command signal is capable of generating the constant output. Note that, although the reference signal is constant for $t \in[0.081,0.4] \mathrm{s}$, the command signal during that time interval is not constant.

\section{B. Hankel ILC}

For Hankel ILC, we define an actuation time interval with $m_{1}=50$ and $m_{2}=81$, giving $m=32$, and the observation time interval by $n_{1}=82$ and $n_{2}=400$, giving $n=319$. The residual vibrations to be suppressed are shown in Fig. $8(\mathrm{a})$. These vibrations are dominated by a resonance of $5.5 \mathrm{~Hz}$ (translation mode), however, errors $e^{1}$ and $e^{2}$ also show higher frequency resonances.

The time weighted system $J_{H}$ for Hankel ILC equals $J_{H}=W_{o} J W_{i}$, with $W_{o}$ from (9) and $W_{i}$ from (20). Based on the singular values of $J_{H}$, Fig. 8(b), three Hankel ILC controllers are designed with $p=12, \gamma=0.5$, and $\beta=1$.

The first controller is based on (12) and (15) without any additional weighting, i.e., with $W=I_{m q_{i}}$. The error signals as function of trial and time are presented in Fig. 9. As expected, the residual vibrations during the observation time interval are suppressed, while the error outside the interval is not compensated for.

The second controller is also based on (12) and (15), however, with a $W$ which minimizes the converged command signal during the actuation time interval: $\min f_{\infty, a c t}^{T} W f_{\infty, a c t}$. Using (16), matrix $W$ is given by:

$$
W:=W_{i}^{T} W_{a c t}^{T} \operatorname{diag}\left(I_{q_{i}}, \cdots, 1.5 I_{q_{i}}\right) W_{a c t} W_{i} .
$$

The diagonal matrix in (21) is used to penalize the command signal amplitudes linearly, with gain 1 for $f_{\infty, a c t}(1)$ up to gain 1.5 for $f_{\infty, a c t}(m)$. Note that in general design of $W$ is based on the designers insight into the problem at hand.

The error results obtained with this second controller are shown in Fig. 10. Though the error $e_{30}$ is slightly larger than $e_{30}$ of Fig. 9, this Hankel ILC controlled system is still very capable of suppressing the residual vibrations.

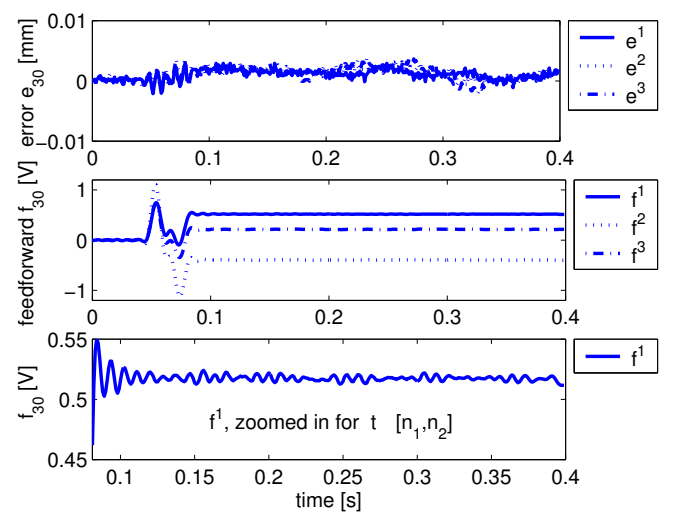

Fig. 7. Standard ILC with additional integrators. Top: Error signal during trial $k=30$. Center: Command signal during trial $k=30$. Bottom: Command signal during trial $k=30$, zoomed in.
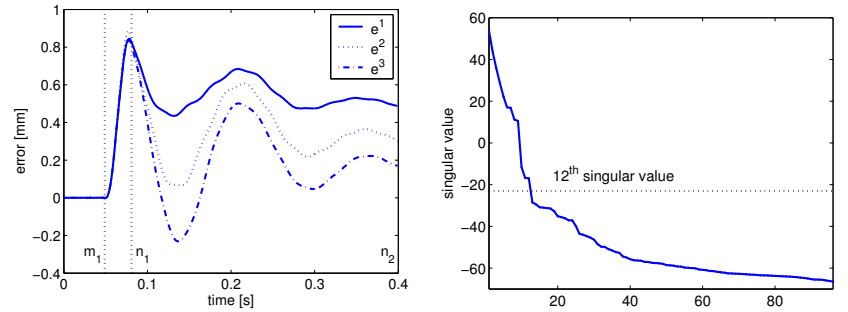

Fig. 8. (a) Residual vibration for the three outputs corresponds to the error signals during $t \in\left[n_{1}, n_{2}\right]$. (b) Singular values of $J_{H}$.
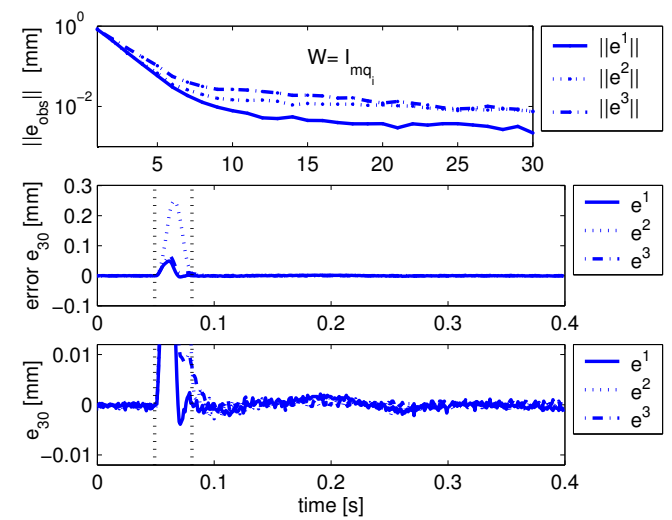

Fig. 9. Hankel ILC with $W=I_{m q_{i}}$. Top: Maximum absolute error as function of the trial. Center: Error signal during trial $k=30$. Bottom: Error signal during trial $k=30$, zoomed in.

Finally, the third controller is based on (12), (14), (17), and (18), with $\Delta_{f}=1$ volt. The error signals corresponding to this controller are given in Fig. 11. The error is again slightly larger than $e_{30}$ of Fig. 9, but still most of the residual vibrations are removed.

Based on the error results, it looks like all three controllers behave approximately equal. This is to be expected, since all three controllers have similar $L_{L}$ and $X_{1}$. The difference between the controllers is related to $Y$, and hence to the command signal forms applied to the system to obtain the above error signals, Fig. 12. The differences in error can

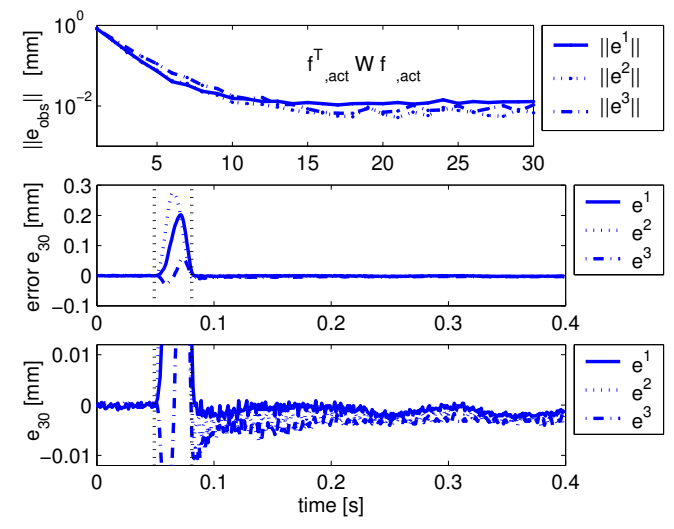

Fig. 10. Hankel ILC with W of (21). Top: Maximum absolute error as function of the trial. Center: Error signal during trial $k=30$. Bottom: Error signal during trial $k=30$, zoomed in. 

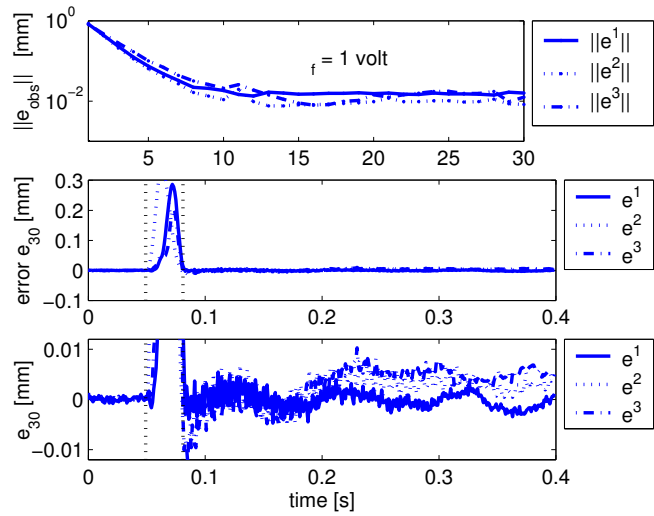

Fig. 11. Hankel ILC with minimized Maximum command signal amplitude. Top: Maximum absolute error as function of the trial. Center: Error signal during trial $k=30$. Bottom: Error signal during trial $k=30$, zoomed in.

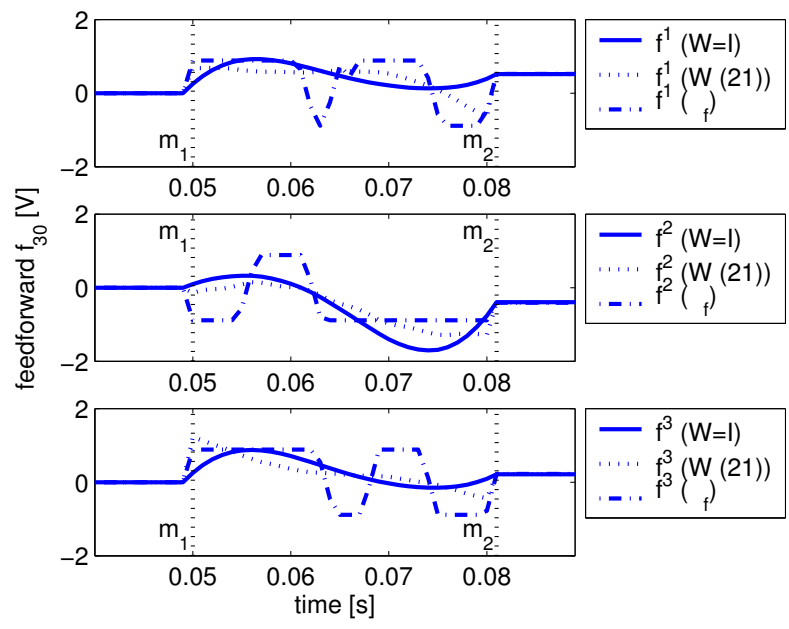

Fig. 12. Command signal $f^{i}, i=1,2,3$, for the three Hankel ILC controllers.

be explained by looking at the smoothness of the different command signals. While the first controller generates relative smooth signals, the command signals of the second and third controller are relatively non-smooth. This non-smoothness causes the excitation of higher frequencies in the system which can not be completely compensated for with $p=12$. The result: relatively high frequent error signals during the observation time interval.

Clearly, the signal form of the different command signals differ during the actuation time interval. After $t=m_{2}$ though, all three command signals are constant and approximately equal (compare with standard ILC, Fig. 7). Furthermore, when comparing the maximum amplitude of the command signals from the first controller with the second controller, a reduction in maximum signal amplitude of $25 \%$ is achieved. The maximum amplitude of the command signals of the third controller is even $48 \%$ smaller than that of the first controller.

\section{CONClusions}

In this paper, we studied residual vibration suppression on a MIMO flexible structure performing a point-to-point motion, based on Hankel ILC. After extensively discussing the different design steps, we experimentally showed that Hankel ILC is capable of suppressing residual vibrations in a relatively complex MIMO flexible structure. The versatility in choice of the actuation and observation time windows turned out to be essential for the successful implementation of Hankel ILC on this flexible structure. Next to vibration suppression results, our experimental results also demonstrated the possibilities of Hankel ILC to manipulate the command signal form.

\section{APPENDIX}

The Singular Value Decomposition of $J_{H} \in \mathbb{R}^{n q_{o} \times m q_{i}}$, with $\operatorname{rank}\left(J_{H}\right)=p$, is given by

$$
\begin{aligned}
& J_{H}=U \Sigma V^{T} \\
& J_{H}=\left[\begin{array}{ll}
U_{1} & U_{2}
\end{array}\right]\left[\begin{array}{cc}
\Sigma_{1} & 0 \\
0 & \Sigma_{2}
\end{array}\right]\left[\begin{array}{l}
V_{1}^{T} \\
V_{2}^{T}
\end{array}\right]=U_{1} \Sigma_{1} V_{1}^{T} \\
& U_{1} \in \mathbb{R}^{n q_{o} \times p}, \quad U_{2} \in \mathbb{R}^{n q_{o} \times n q_{o}-p}, \\
& V_{1} \in \mathbb{R}^{m q_{i} \times p}, \quad V_{2} \in \mathbb{R}^{m q_{i} \times m q_{i}-p}, \\
& \Sigma_{1} \in \mathbb{R}^{p \times p}, \quad \Sigma_{2}=0_{n q_{o}-p \times m q_{i}-p} . \\
& \text { REFERENCES }
\end{aligned}
$$

[1] D. de Roover, "Motion control of a wafer stage," Ph.D. dissertation, Delft University of Technology, 1997, ISBN: 90-47-1562-9.

[2] N. Singer, W. Singhose, and W. Seering, "Comparison of filtering methods for reducing residual vibration," European Journal of Control, vol. 5, pp. 208-218, 1999.

[3] T. Singh and W. Singhose, "Tutorial on input shaping/time delay control of maneuvering flexible structures," in Proc. of the American Control Conference, Anchorage, AK, USA, May 8-10 2002, pp. $1717-$ 1731.

[4] J. van de Wijdeven and O. Bosgra, "Residual vibration suppression using Hankel Iterative Learning Control," in Proc. of the American Control Conference, Minneapolis, MN USA, June 14-16 2006, pp. 1778-1783.

[5] — "Stabilizability, performance, and the choice of actuation and observation time windows in Iterative Learning Control," in Proc. 45th IEEE Conference on Decision and Control, San Diego, CA USA, December 13-15 2006, pp. 5042-5047.

[6] _ - "Residual vibration suppression using Hankel Iterative Learning Control," submitted for publication, 2007.

[7] Z. Bien and J. Xu, Eds., Iterative Learning Control: Analysis, Design, Integration, and Applications. Norwell, MA USA: Kluwer Academic Publishing, 1998, ISBN: 0-7923-8213-7.

[8] K. Moore, Iterative Learning Control for deterministic systems. London: Springer-Verlag, 1993, ISBN: 3-540-19707-9.

[9] J.-X. Xu and Y. Tan, Linear and Nonlinear Iterative Learning Control. Springer-Verlag, 2003, ISBN: 3-540-40173-3.

[10] D. Gorinevsky, "Loop shaping for iterative control of batch processes," IEEE Control Systems Magazine, pp. 55-65, December 2002.

[11] N. Amann, D. Owens, and E. Rogers, "Iterative Learning Control using optimal feedback and feedforward actions," Int. Journal of Control, vol. 65 , no. 2, pp. 277-293, 1996.

[12] S. Gunnarsson and M. Norrlöf, "On the design of ILC algorithms using optimization," Automatica, vol. 37, pp. 2011-2016, 2001.

[13] J. Frueh and M. Phan, "Linear quadratic optimal learning control (LQL)," Int. Journal of Control, vol. 73, no. 10, pp. 832-839, 2000.

[14] B. Dijkstra and O. Bosgra, "Extrapolation of optimal lifted system ILC solution, with application to a waferstage," in Proc. of the American Control Conference, Anchorage, AK USA, May 8-10 2002, pp. 25952600.

[15] J. Hätönen, K. Moore, and D. Owens, "An algebraic approach to Iterative Learning Control," in Proc. of the 2002 IEEE International symposium on intelligent control, Vancouver, Canada, October 27-30 2002 , pp. 37-42.

[16] G. Franklin, J. Powell, and A. Emami-Naeini, Feedback control of dynamic systems. Prentice Hall, 2006, ISBN: 0131499300. 